\title{
广西水稻地方品种核心种质稻瘟病抗性位点全基因组关联分析
}

陈灿 ${ }^{* *}$ 农保选 ${ }^{* *}$ 夏秀忠 张宗琼曾宇冯锐郭 辉
邓国富 李丹婷

广西农业科学院水稻研究所 / 广西水稻遗传育种重点实验室, 广西南宁 530007

摘 要: 稻瘟病是水稻重要病害之一, 严重影响水稻的产量与品质。培育抗性品种是防治稻瘟病最经济、环保的方 式。稻瘟病抗性基因的鉴定与挖掘是开展抗病育种的基础与前提。本课题组前期对 419 份广西水稻地方品种核心种 质进行简化基因组测序, 获得 208,993 个高质量 SNP 标记。本研究采用苗期喷雾接种方法, 研究了该 419 份核心种 质对 7 个稻瘟病生理小种的抗性, 并根据表型和基因型数据, 利用一般线性模型(general linear model, GLM)和混合线 性模型(mixed linear model, MLM)进行全基因组关联分析。2 种模型下共检测到 20 个位点, 其中 GLM 检测到 20 个位 点, MLM 检测到 1 个位点, Chr12_10803913 位点在 2 种模型下都检测到。17 个位点与前人定位的基因/QTLs 重叠, 其 余 3 个是新位点, 分别为 Chr3_18302718、Chr3_18302744 及 Chr5_10379127 位点。在 20 个显著关联位点上下游各 $150 \mathrm{~kb}$ 的基因组区域中共篮选出候选基因 323 个, 初步确定 8 个候选基因与抗病相关, 其中 LOC_Os 12g18360 (Pita)、 $L O C \_O s 12 g 18729$ (Ptr) 为已知克隆的基因, LOC_Os03g32100、LOC_Os03g32180 和 LOC_Os05g18090 为新位点附近 篮选到的候选基因。本研究结果为稻瘟病抗性位点挖掘与稻瘟病相关基因克隆提供了科学依据。

关键词：水稻; 稻瘟病; 全基因组关联分析; 候选基因

\section{Genome-wide association study of blast resistance loci in the core germplasm of rice landraces from Guangxi}

CHEN Can $^{* *}$, NONG Bao-Xuan ${ }^{* *}$, XIA Xiu-Zhong, ZHANG Zong-Qiong, ZENG Yu, FENG Rui, GUO Hui, DENG Guo-Fu, LI Dan-Ting*, and YANG Xing-Hai*

Rice Research Institute, Guangxi Academy of Agricultural Sciences / Guangxi Key Laboratory of Rice Genetics and Breeding, Nanning 530007, Guangxi, China

\begin{abstract}
Blast disease is one of the most important rice diseases, which seriously affects the yield and quality in rice. In general, breeding resistant varieties is the most economical, environmental, and friendly way to control rice blast. Identification and mining of blast resistance genes are the basis and premise of disease resistance breeding. In our previous study, 419 core germplasms from Guangxi rice landraces were sequenced using specific-locus amplified fragment sequencing (SLAF-seq) technology, and 208,993 high-quality SNPs were identified. Spray inoculation at seedling stage was used to evaluate the resistance of the 419 germplasms to 7 strains. According to phenotype and genotype data, genome-wide association study (GWAS) for rice blast was performed using general linear model (GLM) and mixed linear model (MLM). A total of 20 loci were detected under the two models, including 20 loci detected by GLM and 1 locus detected by MLM. Chr12_10803913 locus was detected in both models.
\end{abstract}

\footnotetext{
本研究由中央引导地方科技发展专项(桂科 ZY19183020), 广西创新驱动发展专项(AA17204045-1), 广西自然科学基金项目 (2020GXNSFAA259041，2018GXNSFAA138124，2017GXNSFBA198210)，广西重大科技创新基地开放课题(2018-05-Z06-CX04)和广西 农业科学院发展基金(桂农科 2019Z08)资助。

This study was supported by the Special Fund of Local Science and Technology Development for the Central Guidance (Guike ZY19183020), the Guangxi Special Fund for Innovation-Driven Development (AA17204045-1), the Guangxi Natural Science Fund (2020GXNSFAA259041, 2018GXNSFAA138124, 2017GXNSFBA198210), the Opening Project of Major Science and Technology Innovation Base for Guangxi (2018-05-Z06-CX04), and the Development Fund of Guangxi Academy of Agricultural Sciences (Guinongke 2019Z08).

*通信作者(Corresponding authors): 李丹婷, E-mail: riceg1@163.com; 杨行海, E-mail: yangxinghai514@163.com

** 同等贡献(Contributed equally to this work)

第一作者联系方式: 陈灿, E-mail: chencan129@126.com; 农保选, E-mail: nongbaoxuan88@gxaas.net

Received (收稿日期): 2020-07-12; Accepted (接受日期): 2020-12-01; Published online (网络出版日期): 2020-12-28.

URL: https://kns.cnki.net/kcms/detail/11.1809.S.20201228.1435.008.html
} 
There were 17 loci, overlapping with previously reported genes/QTLs, while the remaining three loci were the first reported, including Chr3_18302718, Chr3_18302744, and Chr5_10379127. A total of 323 candidate genes were screened out in the genomic regions of $150 \mathrm{~kb}$ upstream and downstream of 20 significantly associated loci. Eight candidate genes were preliminarily determined to be related to disease resistance. Among them, both LOC_Os12g18360 (Pita) and LOC_Os12g18729 (Ptr) were known cloned genes, LOC_Os03g32100,LOC_Os03g32180, and LOC_Os05g18090 were selected as candidate genes near the three loci. The results provided the scientific basis for the mining of rice blast resistance loci and gene cloning.

Keywords: rice; blast disease; genome-wide association study (GWAS); candidate genes

稻瘟病是由稻瘟菌(Magnaporthe grisea)引起的 一种水稻毁灭性真菌病害, 严重影响世界水稻种植 区的产量与品质, 从而威胁全球粮食安全 ${ }^{[1-2]}$ 。全球 每年由稻瘟病引起的水稻产量损失约为总量的 $10 \%$ 30\%, 它可以养活至少 6000 万人, 经济价值高 达 660 亿美元 ${ }^{[3]}$ 。化学防治作为一种主要、传统的 病虫害防治手段, 长期使用既污染环境, 也增加经 济成本。种质资源对抗性具有广泛遗传变异, 因此 利用寄主植物自身抗性是防治该病最有效、最经济 和最环保的方法 ${ }^{[4]}$ 。但病原真菌对宿主的适应能力 的频繁突变, 会使得品种抗性在 3 5 年内丧失 ${ }^{[5]}$ 。因 此, 需要不断挖掘鉴定新的稻瘟病基因, 这对于开 展抗病选育有重要的理论与实践意义。

水稻抗性基因包括 2 种类型, 一种是提供种系 特异性抗性的抗性基因(resistance gene, R), 另一种 是控制部分非种系特异性抗性的微效基因, 又称数 量性状位点(quantitative trait locus, QTL)。鉴定抗性 基因(R)/数量性状位点(QTL)及开展抗病机制的研究 对抗病品种的选育尤为重要。水稻稻瘟病抗性基因 挖掘一直是水稻抗病育种的热点问题。迄今为止, 已经鉴定了 110 多个抗稻瘟病基因, 其中克隆了 36 个 $^{[6-8]}$ 。根据克隆基因的编码蛋白类型, 可以分为 5

类。(1) 核苷酸结合位点(nucleotide binding site, NBS)-富含亮氨酸重复序列(leucine rich repeat, LRR)-蛋白(NBS-LRR 或 NLR), 根据 N 端结构域可 分为 2 个子类 TIR (Toll/Interleukin-1 receptor)-NBSLRR 和 CC (coiled-coil)-NBS-LRR。Pi2、Pi9、Pi35、 Pi37、Pi50、Pi56、Piz-t、Pita、Pib、Pid3、Pi-CO39、 Pigm、Pi54、Pid3-A4、Pi54rh、Pik-m 等属于前者, Pi1、 Pi25、Pi36、Pi5、Pit、Pi-k、Pik-p、Pik-e、Pia、

Pi63、Pi64、Pb1、Pish 等属于后者; (2) 凝集素受体 (lectin receptor), 如 Pid2; (3) 富含脯氨酸结构域蛋 白(proline-rich domain proteins), 如 pi21; (4) 富含 Armadillo 重复序列蛋白, 如 Ptr; (5) 富含四肽重复 序列(tetratricopeptide repeats, TPRs)蛋白, 如 $b s r-k 1$ 。 这些编码蛋白类型为稻瘟病候选基因的篮选提供了
重要理论依据。

大多数植物基因/QTL 是通过连锁作图鉴定的, 因此基因/QTL 的检测受到使用的双亲材料的限制, 不能够解释自然界广泛存在的遗传变异。随着高密 度遗传作图技术的发展，基于连锁不平衡的全基因 组关联分析 (genome-wide association analysis, GWAS)已成为自然群体基因/QTL 鉴定的重要工具。 GWAS 克服了利用来自双亲材料的群体进行连锁映 射的缺点。通过与高通量测序、分群分析法(bulked segregant analysis，BSA)等技术手段相结合，GWAS 在检测水稻稻瘟病性状关联位点及篮选候选基因方 面得到了大量快速应用 ${ }^{[9-12]}$ 。如最近 $\mathrm{Li}$ 等 ${ }^{[13]}$ 利用湖 南省 3 个分离菌株和 234 份水稻多样性小组 1 进行 了水稻抗稻瘟病的全基因组关联研究(GWAS), 共 鉴定出 56 个 QTL。其中 1 个 QTL 定位于抗性基因 $P i k$ 位点, 该位点对 3 个分离菌株均具有抗性。抗性 品种基因组序列分析结果表明, 该位点是一个新的 $P i k$ 等位基因, 将其命名为 $P i k x$ 。 $\mathrm{Lu}$ 等 ${ }^{[14]}$ 利用全基 因组关联研究与 RNA 测序分析, 鉴定出 127 个水稻 抗稻瘟病相关位点。此外, 在一个 $200 \mathrm{~kb}$ 的基因组 区域中预测了 2341 个非冗余候选基因, 其中 45 个 基因与抗病相关。

本研究供试材料为 419 份广西地方品种核心种 质, 利用简化基因组测序(specific-locus amplified fragment sequencing, SLAF-seq), 已经获得了高质量 的单核苷酸多态性位点(single nucleotide polymor-

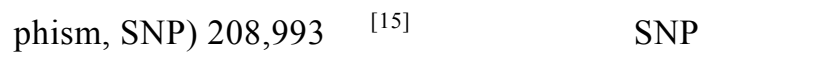
煮食味、糯性、种皮颜色和南方黑条矮缩病等性状 进行了 GWAS 研究 ${ }^{[15-18]}$ 。但利用 GWAS 检测该群体 的稻瘟病抗性位点尚未报道。

为此, 我们拟使用基于 419 份广西地方品种核 心种质的 SNP 数据, 并结合 7 个生理小种的苗期叶 瘟抗性表型数据, 利用 GWAS 挖掘出该群体材料的 稻瘟病关联位点, 并预测显著关联位点附近区域的 候选基因, 为下一步候选基因验证及基因克隆提供 理论依据。 


\section{1 材料与方法}

\section{1 供试材料及菌种}

实验材料来源于广西农业科学院水稻研究所库 存的地方稻种资源核心种质，共计 419 份，包括 330 份籼稻、78 份粳稻及 11 份其他类型品种。丽江新团 黑谷和 Tetep 分别为感病及抗病对照。ZA 9、ZA 13、 $\mathrm{ZB} \mathrm{1、ZB} \mathrm{9、ZB} \mathrm{13、ZC} 3$ 和 ZC 13 等 7 个小种均 由广西农业科学院植物保护研究所提供。水稻材料 及对照品种浸种催芽后播到塑料盘中, 每份材料播 20 粒, 待长至三至五叶龄时(大约 2 3 周)用配制好 的孢子液人工喷雾接种。接种菌液浓度为 $1 \times 10^{5} \sim 2 \times 10^{5}$ 个 $\mathrm{mL}^{-1}$ 的狍子悬浮液 ${ }^{[19]}$, 然后在 $26^{\circ} \mathrm{C}$ 及相对湿度 $95 \%$ 左右的培养室培养 $24 \mathrm{~h}$, 接种大约 $7 \mathrm{~d}$ 后, 根据描述的病斑大小和面积比(diseased leaf area, DLA), 对其进行 0 到 9 分的评分 ${ }^{[20]}$ 。每份材料 鉴定 3 次。0 3 级为抗病(resistance, 用 $\mathrm{R}$ 表示), 4 9 级为感病(susceptibility, 用 $\mathrm{S}$ 表示), 取 3 次平均值为 鉴定结果。利用 SPSS 19 统计分析软件进行描述性 统计及相关作图分析。

\subsection{SLAF 测序和 SNP 基因分型}

在 Illumina Hiseq 2500 系统上进行 SLAF 测序。 利用 BLAT 软件对 clean reads 进行聚类, 得到多态 SLAF 标签。再使用 BWA 软件, 将多态 SLAF 标签 序列比对至日本晴参考基因组上 (http://plants. ensembl.org/Oryza_sativa/Info/Index)。然后利用 GATK and SAM 工具包分析 SNP calling。根据最小 等位基因频率(minor allele frequency, MAF) $>0.05$ 和 完整性>0.5, 共获得 208,993 个 $\mathrm{SNP}^{[15]}$ 。

\section{3 全基因组关联分析的方法}

使用软件 TASSEL V3 对 208,993 个 SNP 基因型 和苗期叶瘟表型数据进行 GWAS 关联分析 ${ }^{[21]}$, 混合
线性模型 MLM 为 $(\mathrm{Q}+\mathrm{K})$ 模型, $\mathrm{Q}$ 为群体结构, $\mathrm{K}$ 为亲 缘系数。采用 MEGA5 软件构建系统发育树。采用 SPAGeDi 软件进行两两亲属关系分析。采用 ADMIXTURE 软件分析种群结构 ${ }^{[15]}$ 。 $P<4.79 \times 10^{-6}$ $(1 / 208,993=4.79 \mathrm{E}-6)$ 被认为具有显著相关性。根据 $\mathrm{R}$ 环境生成 Manhattan 和 Q-Q plot。

\section{4 候选基因的选择}

以日本晴为参考基因组, 基于水稻的连锁不平 衡(linkage disequilibrium, LD)衰减情况, 参考杨行 海等 ${ }^{[16]}$ 研究结果, 选择峰值 SNP 上下游各 $150 \mathrm{~kb}$ 区 间为候选基因区域。所有植物中的抗性基因, 包括 NLR、凝集素受体等被认为是候选基因 ${ }^{[22-23]}$ 。

\section{2 结果与分析}

\section{1 苗期稻瘟病抗性评价}

利用 7 个不同类型的稻瘟病生理小种对 419 份 水稻材料进行苗期喷雾接种, 根据 DLA 评价其抗性, 并对抗性级别进行统计分析。DLA 评估的抗性等级 从“ZC13”的 2.93 到“ZA13”的 5.72, 平均为 4.49, 变 异系数从“ZA13”的 0.24 到“ZC13”的 0.48 , 平均为 0.34 (表 1)。根据抗性级别均值, 可以发现 ZA 种群 毒性最强, 其次是 ZB 种群, ZC 种群较弱, 这与实际 情况相一致, 这说明这 3 类小种对实验材料有很好 的鉴别性。从图 1 可以看出，在接种 7 个不同类型 的稻瘟病生理小种下, 叶瘟抗性级别分布具有较好 的拟合正态分布, 利于开展 GWAS 关联分析。

\section{2 全基因组关联分析}

亲缘关系与群体结构分析表明, 系统发育树聚 集为 2 个主要类群, 其与籼稻、粳稻亚群相一致。 419 份地方种质分为 6 个类型群体结构, 包括籼稻、 粳稻亚类 ${ }^{[15]}$ 。

表 1 不同稻瘟病小种接种下水稻苗期叶瘟抗性级别统计分析

Table 1 Statistical analysis of leaf blast resistance of rice seedlings inoculated with different rice blast strains

\begin{tabular}{lccc}
\hline 小种 & 抗级 & 平均值 \pm 标准差 & $\begin{array}{c}\text { 变异系数 } \\
\text { CV (\%) }\end{array}$ \\
Rtrain & $1-9$ & $5.03 \pm 1.49$ & 29.62 \\
ZA9 & $1-8$ & $5.72 \pm 1.36$ & 23.78 \\
ZA13 & $1-7$ & $4.29 \pm 1.44$ & 33.57 \\
ZB1 & $1-8$ & $4.83 \pm 1.54$ & 31.88 \\
ZB9 & $1-7$ & $3.80 \pm 1.53$ & 40.26 \\
ZB13 & $1-8$ & $4.84 \pm 1.62$ & 33.47 \\
ZC3 & $1-7$ & $2.93 \pm 1.40$ & 47.78 \\
ZC13 & & & \\
\hline
\end{tabular}



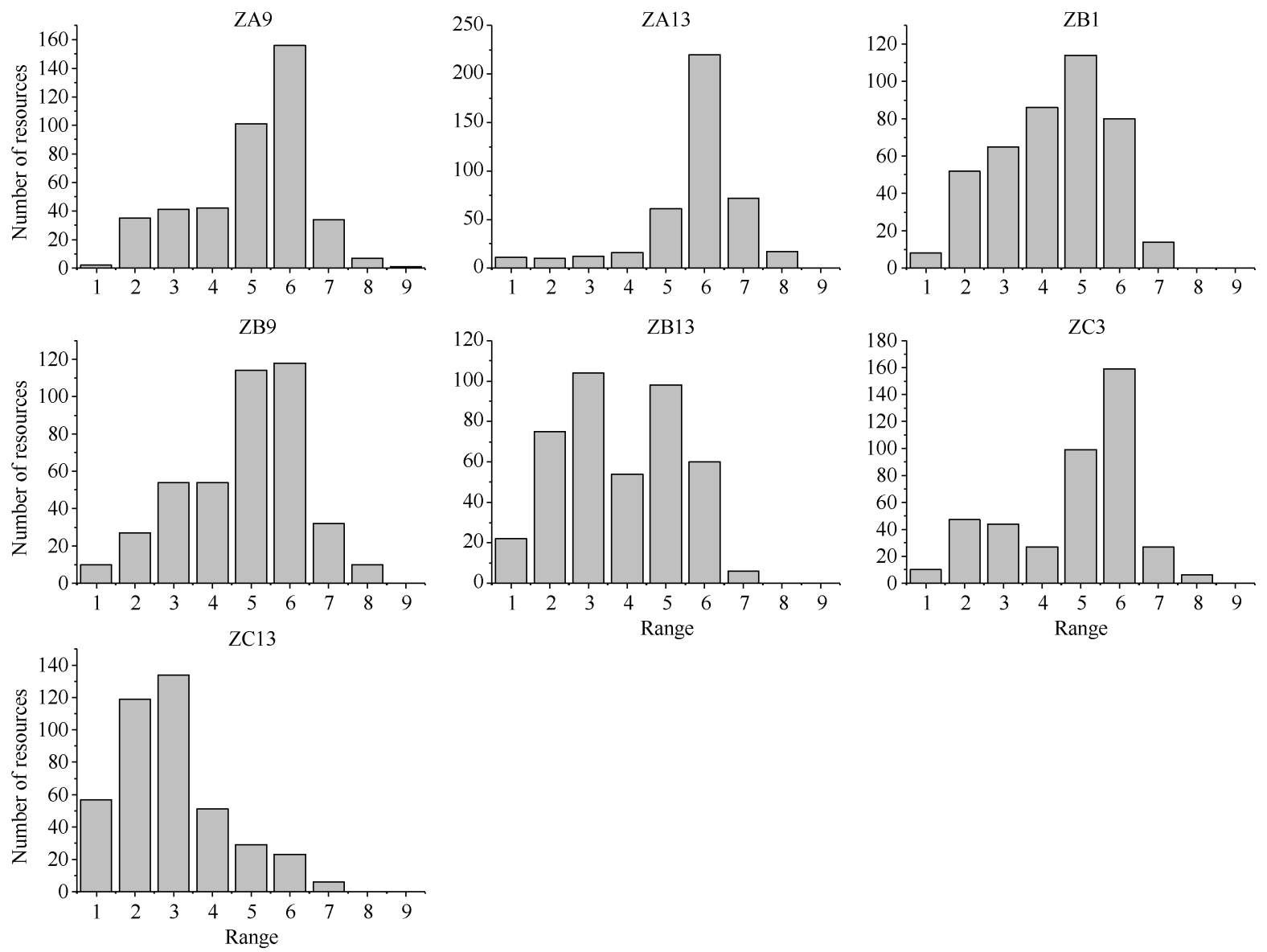

图 1 关联群体在 7 个不同稻瘟病生理小种接种下的叶瘟抗性级别分布

Fig. 1 Distribution of resistance levels of leaf blast among the associated population inoculated with seven strains

在 $P<4.79 \times 10^{-6}(4.79 \mathrm{E}-6)$ 水平下，使用一般线 性模型(GLM)共检测到20个稻瘟病相关 SNP (图 2-A C 和表2), 其中位点 10,803,913在 MLM 下也 被检测到, 3 个位点为新位点, 分别为 3 号染色体上 的 18,302,718、18,302,744 位点 (Chr3_18302718, Chr3_18302744) 及 5 号染色体上 10,379,127位点 (Chr5_10379127), 其他 17个位点附近均有已知定 位基因 $/ \mathrm{QTL}$ 。在接种的 7 个小种中, 只有 3 个小种 关联到显著性位点, 平均每个小种关联到6.7个位 点。ZC3 关联到最多的位点, 共16个, ZC13、ZB9 各关联到 2 个位点。从关联位点在染色体上的分布 来看, 12 号染色体关联的位点最多, 为 13 个, 其次 是 1 号和 3 号染色体, 分别为 3 个和 2 个, 5 号和 8 号染 色体最少, 均为 1 个。

在 $P<4.79 \times 10^{-6}(4.79 \mathrm{E}-6)$ 水平下, 使用混合线性 模型(MLM)仅检测到 1 个稻瘟病相关 SNP (图 2-D 和 表 2), 该位点 $10,803,913$ 位于 12 号染色体 (Chr12_10803913)。

\section{3 候选基因分析}

根据水稻基因组注释 (http://rice.plantbiology. msu.edu/)及 LD 衰退水平, 在 20 个关联位点 $300 \mathrm{~kb}$ 的基因组区域中共鉴定出候选基因 323 个。根据已 知克隆稻瘟病抗性基因的编码蛋白类型、候选基因 功能注释信息及利用植物比较基因组学资源库 (https://phytozome.Jgi.doe.gov/pz/portal.html)蛋白序 列同源比对分析, 初步篮选到 8 个候选基因与稻瘟 病相关, 其中 LOC_Os12g18360 (Pita)、LOC_ Os12g18729 (Ptr)为已知克隆基因(表 3)。本研究鉴 定到的一些 SNP 位点与这 2 个克隆基因距离非常接 近。如 12 号染色体上的位点 $10,629,609(P=6.56 \mathrm{E}-08)$ 与 Pita $^{[24-25]}(10,606,359 \sim 10,612,068)$ 相距仅约 $17.5 \mathrm{~kb}$; 该染色体上的另一个位点 $10,816,338(P=8.01 \mathrm{E}-09)$ 与 $P t r^{[26]}(10,822,534 \sim 10,833,768)$ 相距仅约 $6.2 \mathrm{~kb}$ 。 LOC_Os01g14550和 LOC_Os01g14590功能注释为病 理相关蛋白, LOC_Os12g18374 编码含 NB-ARC 结构 蛋白。说明这 3 个基因可能是抗病候选基因。 

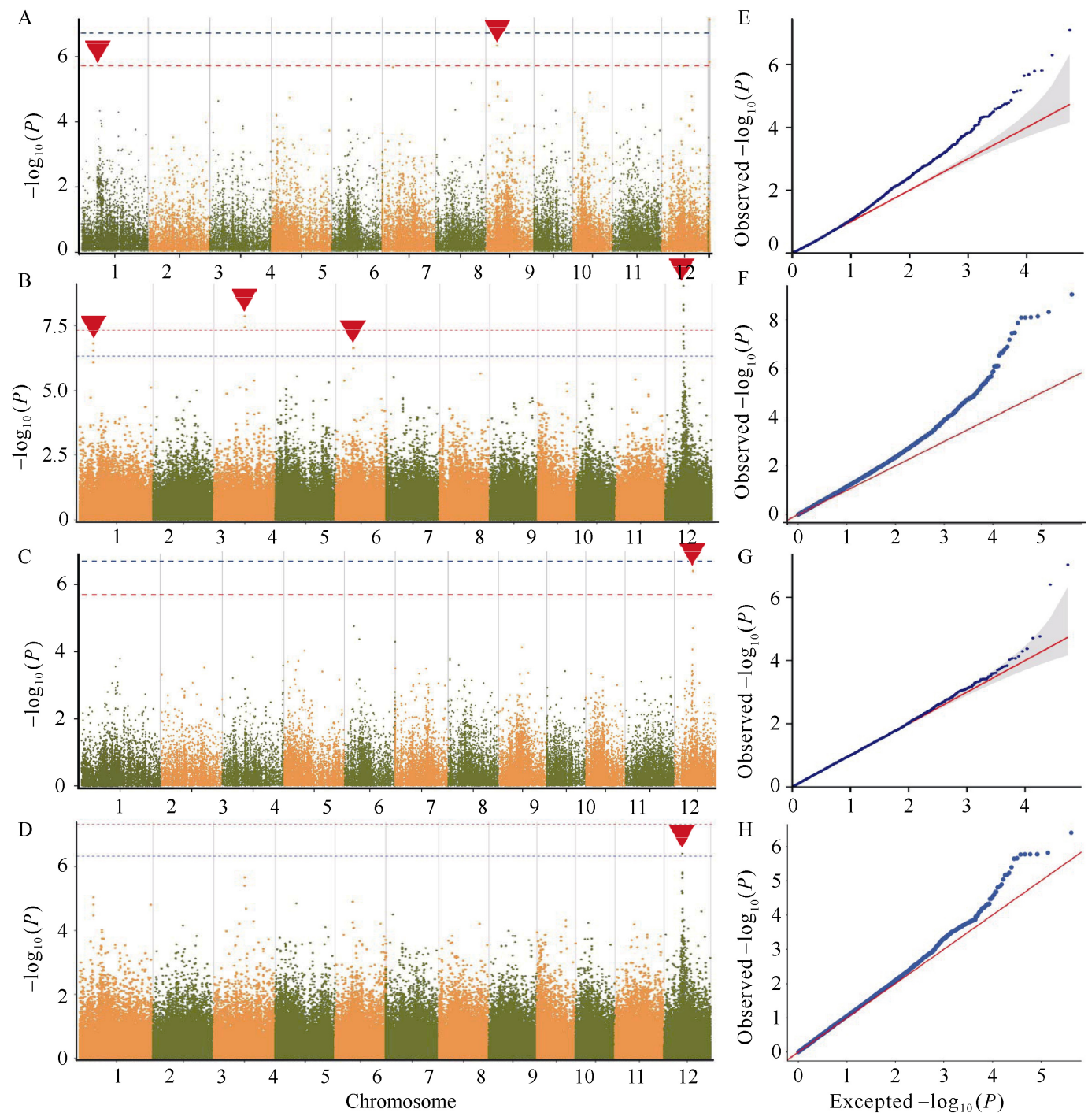

图 23 个菌种下水稻稻瘟病抗性的全基因组关联研究

Fig. 2 Genome-wide association study of rice blast resistance to three strains

A、B、C 分别表示 GLM 模型 ZB9、ZC3、ZC13 的曼哈顿图。D 表示 MLM 模型 ZC3 的曼哈顿图。E、F、G 分别表示 GLM 模型 ZB9、 $\mathrm{ZC} 3 、 \mathrm{ZC} 13$ 的 QQ 图。 $\mathrm{H}$ 表示 MLM 模型 $\mathrm{ZC} 3$ 的 QQ 图。图中实心倒三角形为本文中显著性关联位点。

A, B, and C represent Manhattan plot of GLM model ZB9, ZC3, and ZC13, respectively. D represents Manhattan plot ZC3 in MLM model. D, E, and F represent Quantitle-Quantitle plot of GLM model ZB9, ZC3, and ZC13, respectively. H represents Quantitle-Quantitle plot ZC3 in MLM model. The solid inverse triangle in the figure is the significant correlation point in this study.

表 2 水稻稻瘟病显著关联的 SNP 位点及已定位的基因/QTL

Table 2 SNP locus of significant association of rice blast and located genes/QTLs

\begin{tabular}{|c|c|c|c|c|c|c|c|}
\hline $\begin{array}{l}\text { 小种 } \\
\text { Strain }\end{array}$ & $\begin{array}{c}\text { 染色体 } \\
\text { Chr. }\end{array}$ & $\begin{array}{c}\text { 位置 } \\
\text { Position }\end{array}$ & $\begin{array}{c}P \text { 值 } \\
P \text {-value }\end{array}$ & $\begin{array}{c}\text { 上游位点 } \\
\text { Upstream loci }\end{array}$ & $\begin{array}{c}\text { 下游位点 } \\
\text { Downstream loci }\end{array}$ & $\begin{array}{c}\text { 模型 } \\
\text { Model } \\
\end{array}$ & $\begin{array}{c}\text { 已知基因/QTL } \\
\text { Known genes/QTLs }\end{array}$ \\
\hline ZB9 & 1 & $10,443,043$ & $1.63 \mathrm{E}-06$ & $10,293,043$ & $10,593,043$ & GLM & $P i-h 2(t)$ \\
\hline $\mathrm{ZC} 3$ & 1 & $8,116,727$ & $1.54 \mathrm{E}-07$ & $7,966,727$ & $8,266,727$ & GLM & $P i-s j 9$ \\
\hline $\mathrm{ZC} 3$ & 1 & $8,116,887$ & $2.91 \mathrm{E}-07$ & $7,966,887$ & $8,266,887$ & GLM & $P i-s j 9$ \\
\hline $\mathrm{ZC} 3$ & 3 & $18,302,718$ & $3.60 \mathrm{E}-08$ & $18,152,718$ & $18,452,718$ & GLM & \\
\hline $\mathrm{ZC} 3$ & 3 & $18,302,744$ & $1.35 \mathrm{E}-08$ & $18,152,744$ & $18,452,744$ & GLM & \\
\hline $\mathrm{ZC} 3$ & 5 & $10,379,127$ & $2.32 \mathrm{E}-07$ & $10,229,127$ & $10,529,127$ & GLM & \\
\hline ZB9 & 8 & $6,776,078$ & 4.99E-07 & $6,626,078$ & $6,926,078$ & GLM & Pizh, Pi42 \\
\hline
\end{tabular}


(续表 2)

\begin{tabular}{|c|c|c|c|c|c|c|c|}
\hline $\begin{array}{l}\text { 小种 } \\
\text { Strain }\end{array}$ & $\begin{array}{c}\text { 染色体 } \\
\text { Chr. }\end{array}$ & $\begin{array}{c}\text { 位置 } \\
\text { Position }\end{array}$ & $\begin{array}{c}P \text { 值 } \\
P \text {-value }\end{array}$ & $\begin{array}{c}\text { 上游位点 } \\
\text { Upstream loci }\end{array}$ & $\begin{array}{c}\text { 下游位点 } \\
\text { Downstream loci }\end{array}$ & $\begin{array}{c}\text { 模型 } \\
\text { Model }\end{array}$ & $\begin{array}{c}\text { 已知基因/QTL } \\
\text { Known genes/QTLs }\end{array}$ \\
\hline ZC13 & 12 & $10,917,077$ & $9.44 \mathrm{E}-08$ & $10,767,077$ & $11,067,077$ & GLM & Pi12, Pi157, Pi19(t), Pi20, \\
\hline $\mathrm{ZC} 13$ & 12 & $10,919,541$ & $3.98 \mathrm{E}-07$ & $10,769,541$ & $11,069,541$ & GLM & Pi31(t), Pi42(t), Pi6(t), \\
\hline $\mathrm{ZC} 3$ & 12 & $10,629,609$ & $6.56 \mathrm{E}-08$ & $10,479,609$ & $107,79,609$ & GLM & $\begin{array}{l}\text { Pita, Pita2, Pi67, Pi39(t), } \\
\text { Pi58(t), Pi57, Ptr }\end{array}$ \\
\hline $\mathrm{ZC} 3$ & 12 & $10,796,961$ & $1.84 \mathrm{E}-07$ & $10,646,961$ & $10,946,961$ & GLM & \\
\hline $\mathrm{ZC} 3$ & 12 & $10,801,871$ & $1.30 \mathrm{E}-07$ & $10,651,871$ & $10,951,871$ & GLM & \\
\hline $\mathrm{ZC} 3$ & 12 & $10,803,744$ & 7.39E-09 & $10,653,744$ & $10,953,744$ & GLM & \\
\hline $\mathrm{ZC} 3$ & 12 & $10,803,791$ & $3.45 \mathrm{E}-08$ & $10,653,791$ & $10,953,791$ & GLM & \\
\hline $\mathrm{ZC} 3$ & 12 & $10,803,913$ & $9.20 \mathrm{E}-10$ & $10,653,913$ & $10,953,913$ & GLM & \\
\hline $\mathrm{ZC} 3$ & 12 & $10,816,142$ & 4.90E-09 & $10,666,142$ & $10,966,142$ & GLM & \\
\hline $\mathrm{ZC} 3$ & 12 & $10,816,145$ & $8.24 \mathrm{E}-09$ & $10,666,145$ & $10,966,145$ & GLM & \\
\hline $\mathrm{ZC} 3$ & 12 & $10,816,166$ & $8.24 \mathrm{E}-09$ & $10,666,166$ & $10,966,166$ & GLM & \\
\hline $\mathrm{ZC} 3$ & 12 & $10,816,338$ & $8.01 \mathrm{E}-09$ & $10,666,338$ & $10,966,338$ & GLM & \\
\hline $\mathrm{ZC} 3$ & 12 & $10,926,790$ & $2.35 \mathrm{E}-07$ & $10,776,790$ & $11,076,790$ & GLM & \\
\hline $\mathrm{ZC} 3$ & 12 & $10,803,913$ & $3.93 \mathrm{E}-07$ & $10,653,913$ & $10,953,913$ & MLM & \\
\hline
\end{tabular}

通过蛋白序列同源比对, 在 3 个新的关联位点 附近篮选到 3 个可能与稻瘟病相关的候选基因, 分 别 为 LOC_Os03g32100、LOC_Os03g32180 和 LOC_Os05g18090。LOC_Os03g32100 编码蛋白与玉 米的 2 个基因 GRMZM2G09049、GRMZM2G151649 编码产物相似度为 $66.8 \% 、 69.0 \%$, 这 2 个基因都与 ARM 重复超家族蛋白相关, 推测此基因可能属于 含 ARM 重复序列蛋白抗病类型。LOC_Os03g32180 编码蛋白与菠萝的 1 个基因 Aco021559.1 编码产 物相似度为 $60.8 \%$ 。该基因编码富亮氨酸重复受 体蛋白激酶家族蛋白, 推测此基因可能属于受体 蛋白激酶抗病类型。LOC_Os05g18090 编码蛋白 与玉米的 1 个基因 GRMZM2G151567 编码产物相 似度为 $57.9 \%$ 。该基因编码富亮氨酸重复跨膜蛋
白激酶，推测此基因可能属于受体蛋白激酶抗病 类型。

\section{3 讨论}

GWAS 已广泛应用于植物各种性状的基因位点 挖掘。在稻瘟病抗性基因方面, 前人已经开展了大 量的研究工作, 并关联到一些与已知基因/QTL 相重 叠的显著性关联位点 ${ }^{[9-14]}$ 。这些基因/QTL 主要是 Pita、Ptr、Pi-6 和 Pi42 等。本研究也在这些基因/QTL 附近关联到一些显著性位点，这说明这些基因/QTL 可能广泛存在于自然群体材料中。

在 1 号染色体上, 本研究共鉴定出 3 个显著关 联位点, 这些位点与该染色体上的 2 个基因相重叠 (图 3-A), 分别是 $P i-s j 9^{[27]}(7.86 \mathrm{Mb} 4.97 \mathrm{Mb}$ )和

表 3 候选基因信息

Table 3 Information of candidate genes

\begin{tabular}{ccl}
\hline $\begin{array}{c}\text { 基因名称 } \\
\text { Gene name }\end{array}$ & $\begin{array}{c}\text { 物理位置 } \\
\text { Physical position }\end{array}$ & \multicolumn{1}{c}{$\begin{array}{c}\text { 基因注释 } \\
\text { Gene annotation }\end{array}$} \\
\hline LOC_Os01g14550 & $8,159,849-8,161,554$ & Pathogen-related protein, putative, expressed \\
LOC_Os01g14590 & $8,175,375-8,177,889$ & Pathogen-related protein, putative, expressed \\
LOC_Os03g32100 & $18,367,156-18,368,378$ & Spotted leaf 11, putative, expressed \\
LOC_Os03g32180 & $18,410,063-18,411,482$ & Polygalacturonase inhibitor 1 precursor, putative, expressed \\
LOC_Os05g18090 & $10,404,235-10,407,571$ & SHR5-receptor-like kinase, putative, expressed \\
LOC_Os12g18360 & $10,606,359-10,612,068$ & NB-ARC domain containing protein, expressed \\
LOC_Os12g18374 & $10,624,037-10,633,368$ & NB-ARC domain containing protein, expressed \\
LOC_Os12g18729 & $10,822,534-10,833,768$ & Expressed protein \\
\hline
\end{tabular}


$P i-h 2(t)^{[28]}(8.41 \mathrm{Mb} \sim 18.83 \mathrm{Mb})$ 。在 8 号染色体上, 本 研究共鉴定出 1 个显著关联位点, 该位点与该染色 体上的 2 个基因相重叠, 分别是 $P i-z h^{[29]}$ (4.37 $\mathrm{Mb} 21.01 \mathrm{Mb})$ 和 $P i 42^{[30]}(5.11 \mathrm{Mb} \sim 6.76 \mathrm{Mb})$ 。在 12 号染色体上, 本研究共鉴定出 13 个显著关联位点, 这些位点与该染色体上的 14 个基因相重叠或位于 其附近(图 3-B)其分别为: $P i t a^{[24-25]}(10.60 \mathrm{Mb} \sim 10.60$ $\mathrm{Mb}) 、 P t r^{[26]}(10.82 \mathrm{Mb} \sim 10.83 \mathrm{Mb}) 、 P i 31(t)^{[31]}(7.73$ $\mathrm{Mb} \sim 11.91 \mathrm{Mb}) 、 P i 12^{[32]}(6.98 \mathrm{Mb} \sim 15.12 \mathrm{Mb}) 、 P i 157^{[33]}$ $(8.82 \mathrm{Mb} \sim 18.05 \mathrm{Mb}) 、 P i 19(t)^{[34]}(10.73 \mathrm{Mb} \sim 12.04$ $\mathrm{Mb}) 、 P i 20^{[35]}(6.98 \mathrm{Mb} \sim 10.60 \mathrm{Mb}) 、 P i 42(t)^{[36]}(9.36$ $\mathrm{Mb} \sim 12.24 \mathrm{Mb}) 、 P i 6(t)^{[37]}(4.05 \mathrm{Mb} \sim 18.86 \mathrm{Mb})$ 、 Pita2 $^{[38]}(10.07 \mathrm{Mb} \sim 13.21 \mathrm{Mb}) 、 P i 67^{[39]}(10.60 \mathrm{Mb} \sim$
$12.63 \mathrm{Mb}) 、 P i 39(t)^{[40]}(10.61 \mathrm{Mb} \sim 10.65 \mathrm{Mb}) 、 P i 58(t)^{[41]}$ $(7.46 \mathrm{Mb} \sim 11.26 \mathrm{Mb})$ 和 $P i 57^{[42]}(10.79 \mathrm{Mb} \sim 10.85 \mathrm{Mb})$ 。 以上说明本研究群体可能含有上述基因。

在 3 号染色体及 5 号染色体上, 本研究分别鉴定出 2 个(Chr3_18302718、Chr3_18302744)和 1 个(Chr5 10379127)显著关联位点, 这些位点都与染色体上已经 定位的基因(pi66 $(t)^{[43]}(22.92 \mathrm{Mb} 27.89 \mathrm{Mb})$ 和 Pi6 $8^{[44]}$ $(9.33 \mathrm{Mb} \sim 9.78 \mathrm{Mb})$ 在 3 号染色体上; $P i 10^{[45]}(14.52$ $\mathrm{Mb} \sim 18.85 \mathrm{Mb}) 、 P i 23^{[46]}(10.75 \mathrm{Mb} \sim 19.17 \mathrm{Mb})$ 和 $P i 26(t)^{[31]}$ (2.06 Mb 2.76 Mb)在 5 号染色体上)不重叠。据此推测, 这些位点附近可能含有新的抗稻瘟病基因。

进一步候选基因分析表明, 初步篮选到了 8 个 与稻瘟病相关的候选基因, 其中 LOC_Os $12 \mathrm{~g} 18360$

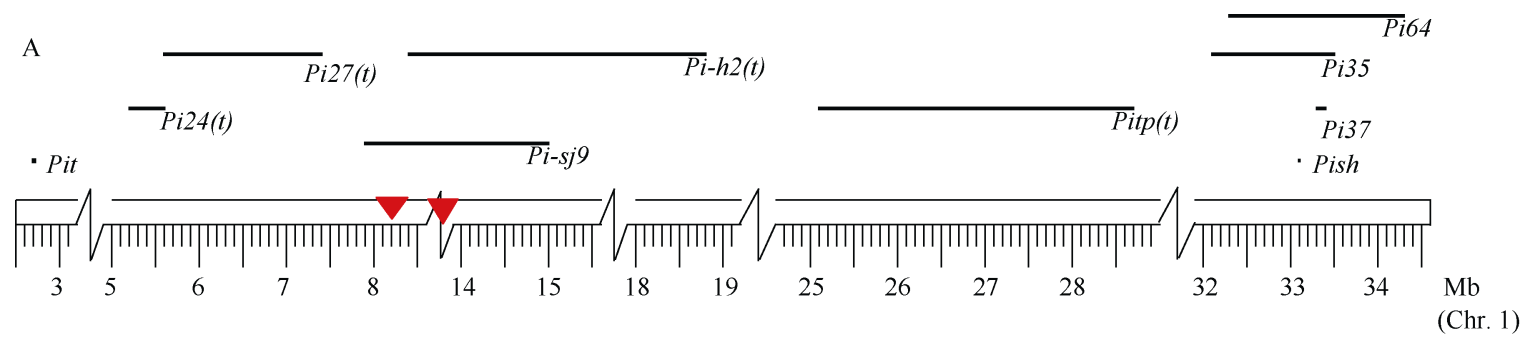

B

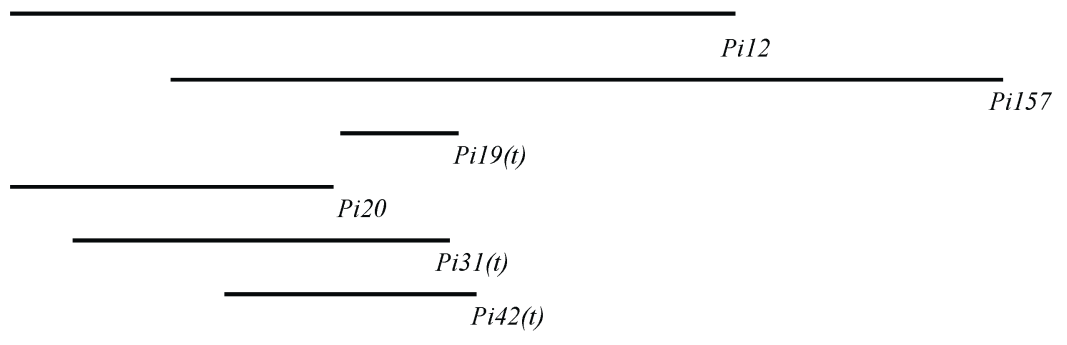

$\operatorname{Pi6}(t)$
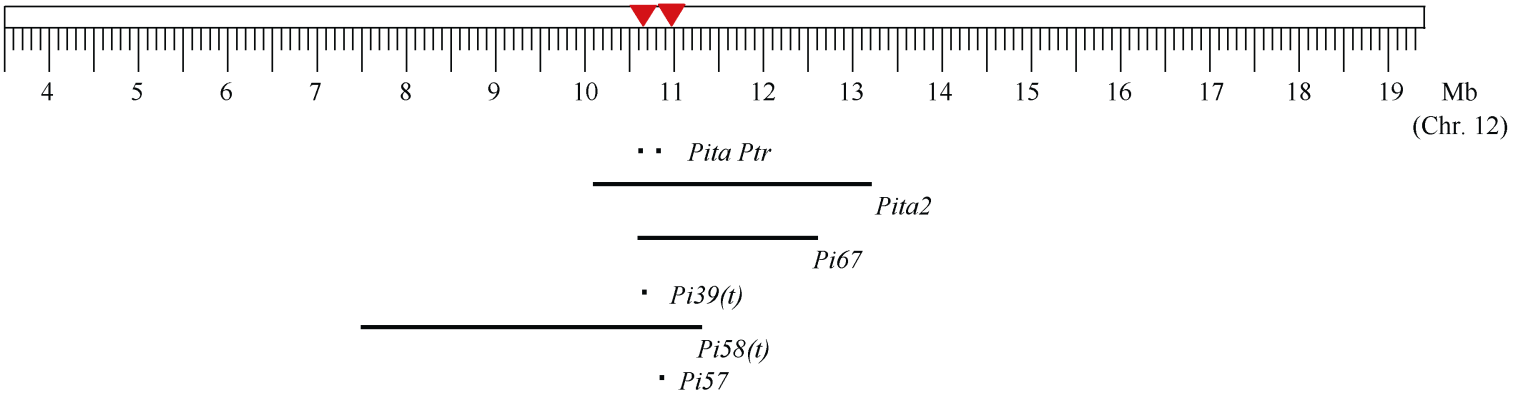

图 3 已定位的稻瘟病抗性基因与关联位点

Fig. 3 Identified blast resistance genes and their associated loci

倒三角形所指关联位点或关联位点所在区域。A: 1 号染色体上已定位的稻瘟病基因; B: 12 号染色体上与显著关联位点区域重叠的稻 瘟病基因。

An inverted triangle refers to the region of an association point or associated bit. A: the rice blast genes located on chromosome 1; B: the rice blast genes on chromosome 12 overlapped with the significantly associated locus region. 
(Pita)、LOC_Os12g18729 (Ptr) 为已知克隆基因。 LOC_Os12g18374 也被其他研究者认为是稻瘟病相 关的候选基因 ${ }^{[47]}$, 这与本研究结果相一致。 LOC_Os03g32100、LOC_Os03g32180 和 LOC_ $O s 05 g 18090$ 为新关联位点附近篮选到的候选基因, 其中 LOC_Os03g 32100 在接种稻瘟菌后基因表达量 显著上调 ${ }^{[48]}$ 。其他候选基因如 LOC_OsO3g32230 和 LOC_Os03g32220 在接种稻瘟菌后基因表达量也显 著上调 ${ }^{[48-49]}$, 其中 LOC_Os03g32230 (bsr-d1/ZOS312)是一个非小种特异性的 $\mathrm{C}_{2} \mathrm{H}_{2}$ 转录因子, 协调减 弱过氧化氢的降解, 表现出对稻瘟病的广谱抗性 ${ }^{[50]}$ 。 本研究发现, 虽然 LOC_Os03g32230 在关联位点 Chr3_18302718 和 Chr3_18302744 的区间内, 但是该 位点仅在接种 ZC3 小种后鉴定到, 即该位点上的候 选基因不具有广谱抗性, 故不含有 bsr-d1/ZOS3-12。 以上候选基因是否在本研究中参与稻瘟病抗性过程, 还需要作进一步的实时定量 PCR 验证。

\section{4 结论}

基于 GLM 和 MLM 模型的 GWAS 结果，共检测 到 20 个与稻瘟病抗性显著相关的 SNP 位点, 其中 17 个位点与前人定位的基因/QTLs 重叠, 其余 3 个 是新位点。在关联位点区域初步确定 8 个候选基因 与抗病相关, 包括 2 个克隆的基因 $L O C_{-}$ Os12g18360(Pita)、LOC_Os12g18729 (Ptr)及3 个新 位点附近篮选到的基因 LOC_Os03g32100、LOC Os03g32180 和LOC_Os05g18090。

\section{References}

[1] Ashkani S, Rafii M Y, Shabanimofrad M, Miah G, Sahebi M, Azizi P, Tanweer F A, Akhtar M S, Nasehi A. Molecular breeding strategy and challenges towards the improvement of blast disease resistance in rice crop. Front Plant Sci, 2015, 6: 886.

[2] Sakulkoo W, Osés-Ruiz M, Garcia E O, Soanes D M, Littlejohn G R, Hacker C, Correia A, Valent B, Talbot N J. A single fungal MAP kinase controls plant cell-to-cell invasion by the rice blast fungus. Science, 2018, 359: 1399-1403.

[3] Scheuermann K K, Raimondi J V, Marschalek R, Andrade A D, Wickert E. The Molecular Basis of Plant Genetic Diversity. Shanghai: InTech China, 2012. pp 331-356.

[4] Manandhar H K, Lyngs Jorgensen H J, Mathur S B, Smedegaard-Peterson V. Suppression of rice blast by preinoculation with avirulent Pyricularia oryzae and the nonrice pathogen Bipolaris sorokiniana. Phytopathology, 1998, 88: 735-739.

[5] Zeigler R S, Leong S A, Teng P S. Rice Blast Disease. Wallingford: CAB International, 1994. pp 626.

[6] 曹妮, 陈渊, 季芝娟, 曾宇翔, 杨长登, 梁燕. 水稻抗稻瘟病 分子机制研究进展. 中国水稻科学, 2019, 33: 489-498.
Cao N, Chen Y, Ji Z J, Zeng Y X, Yang C D, Liang Y. Recent progress in molecular mechanism of rice blast resistance. Chin $J$ Rice Sci, 2019, 33: 489-498 (in Chinese with English abstract).

[7] Zheng C Q, Jiang N, Zhao X H, Yan T Z, Fu J, Li Y F, Wu Z X, Hu X C, Bai Z N, Liu T G, Xiao G, Zhou Y B, Chen L B, Wang K, Yang Y Z. Identification of the blast resistance gene Picl( $(t)$ from Chaling common wild rice (Oryza rufipogon Griff.). J Phytopathol, 2020, 168: 211-219.

[8] Kalia S, Rathour R. Current status on mapping of genes for resistance to leaf- and neck-blast disease in rice. 3 Biotech, 2019, 9: 209.

[9] Mgonja E M, Park C H, Kang H X, Balimponya E G, Opiyo S, Bellizzi M, Mutiga S K, Rotich F, Ganeshan V D, Mabagala R, Sneller C, Correll J, Zhou B, Talbot N J, Mitchell T K, Wang G L. Genotyping-by-sequencing-based genetic analysis of African rice cultivars and association mapping of blast resistance genes against Magnaporthe oryzae populations in Africa. Phytopathology, 2017, 107: 1039-1046.

[10] Wang C H, Yang Y L, Yuan X P, Xu Q, Feng Y, Yu H Y, Wang Y $\mathrm{P}$, Wei X H. Genome-wide association study of blast resistance in indica rice. BMC Plant Biol, 2014, 14: 311-321.

[11] Kang H X, Wang Y, Peng S S, Zhang Y L, Xiao Y H, Wan D, Qu S H, Li Z Q, Yan S Y, Wang Z L, Liu W D, Ning Y S, Korniliev P, Leung H, Mezey J, Mccouch S R, Wang G L. Dissection of the genetic architecture of rice resistance to the blast fungus Magnaporthe oryzae. Mol Plant Pathol, 2016, 17: 959-972.

[12] Lin H A, Chen S Y, Chang F Y, Tung C W, Chen Y C, Shen W C, Chen R S, Wu C W, Chung C L. Genome-wide association study of rice genes and loci conferring resistance to Magnaporthe oryzae isolates from Taiwan. Bot Stud, 2018, 59: 32.

[13] Li C G, Wang D, Peng S S, Chen Y, Su P, Chen J B, Zheng L M, Tan X Q, Liu J L, Xiao Y H, Kang H X, Zhang D Y, Wang G L, Liu Y. Genome-wide association mapping of resistance against rice blast strains in South China and identification of a new Pik allele. Rice, 2019, 12: 47.

[14] Lu Q, Wang C H, Niu X J, Zhang M C, Xu Q, Feng Y, Yang Y L, Wang S, Yuan X P, Yu H Y, Wang Y P, Wei X H. Detecting novel loci underlying rice blast resistance by integrating a genome-wide association study and RNA sequencing. Mol Breed, 2019, 39: 81.

[15] Yang X H, Xia X Z, Zeng Y, Nong B X, Zhang Z Q, Wu Y Y, Xiong F Q, Zhang Y X, Liang H F, Deng G F, Li D T. Identification of candidate genes for gelatinization temperature, gel consistency and pericarp color by GWAS in rice based on SLAF-sequencing. PLoS One, 2018, 13: e0196690.

[16] 杨行海, 农保选, 夏秀忠, 张宗琼, 曾宇, 刘开强, 邓国富, 李 丹婷. 水稻糯性相关基因的全基因组关联分析. 植物学报, 2016, 51: 737-742.

Yang X H, Nong B X, Xia X Z, Zhang Z Q, Zeng Y, Liu K Q, Deng G F, Li D T. Genome-wide association study of genes related to waxiness in Oryza sativa. Chin Bull Bot, 2016, 51: 737-742 (in Chinese with English abstract).

[17] 杨行海, 农保选, 夏秀忠, 张宗琼, 曾宇, 刘开强, 邓国富, 李 丹婷. 广西地方稻种资源核心种质红色种皮全基因组关联分 析及鉴定两个新的 $R c$ 等位基因. 分子植物育种, 2017, 15: 1-6. Yang X H, Nong B X, Xia X Z, Zhang Z Q, Zeng Y, Liu K Q, Deng G F, Li D T. Validation of the red pericarp gene from 419 
rice landrace core collection in Guangxi using genome-wide association study and discovery of two novel $R c$ alleles. $\mathrm{Mol}$ Plant Breed, 2017, 15: 1-6 (in Chinese with English abstract).

[18] 农保选, 秦碧霞, 夏秀忠, 杨行海, 张宗琼, 曾宇, 刘驰, 蔡健 和, 谢慧婷, 崔丽贤, 罗群昌, 邓国富, 刘丕庆, 李丹婷. 南方 水稻黑条矮缩病苗期抗性的全基因组关联分析. 分子植物育 种, 2019, 17: 1069-1079.

Nong B X, Qin B X, Xia X Z, Yang X H, Zhang Z Q, Zeng Y, Liu C, Cai J H, Xie H T, Cui L X, Luo Q C, Deng G F, Liu P Q, Li D

T. Genome-wide association study of seedling resistance of southern rice black-streaked dwarf virus. Mol Plant Breed, 2019, 17: 1069-1079 (in Chinese with English abstract).

[19] Park C H, Chen S B, Shirsekar G, Zhou B, Khang C H, Songkumarn P, Afzal A J, Ning Y S, Wang R Y, Bellizzi M, Valent B, Wang G L. The Magnaporthe oryzae effector AvrPiz-t targets the RING E3 ubiquitin ligase APIP6 to suppress pathogen-associated molecular pattern-triggered immunity in rice. Plant Cell, 2012, 24: 4748-4762.

[20] IRRI (International Rice Research Institute). Standard Evaluation System for Rice. Philippines: International Rice Research Institute, Manila, Philippines. 1996. pp 17-18.

[21] Zhang Z W, Ersoz E, Lai C Q, Todhunter R J, Tiwari H K, Gore M A, Bradbury P J, Yu J M, Arnett D K, Ordovas J M, Buckler E S. Mixed linear model approach adapted for genome-wide association studies. Nat Genet, 2010, 42: 355-360.

[22] Liu W D, Liu J L, Triplett L, Leach J E, Wang G L. Novel insights into rice innate immunity against bacterial and fungal pathogens. Annu Rev Phytopathol, 2014, 52: 213-241.

[23] Kourelis J, van der Hoorn R A L. Defended to the nines: 25 years of resistance gene cloning identifies nine mechanisms for R protein function. Plant Cell, 2018, 30: 285-299.

[24] Orbach M J, Farrall L, Sweigard J A, Chumley F G, Valent B. A telomeric avirulence gene determines efficacy for the rice blast resistance gene Pi-ta. Plant Cell, 2000, 12: 2019-2032.

[25] Bryan G T, Wu K S, Farrall L, Jia Y L, Hershey H P, McAdams S A, Faulk K N, Donaldson G K, Tarchini R, Valent B. A single amino acid difference distinguishes resistant and susceptible alleles of the rice blast resistance gene Pi-ta. Plant Cell, 2000, 12: 2033-2045.

[26] Zhao H J, Wang X Y, Jia Y L, Minkenberg B, Wheatley M, Fan J B, Jia M H, Famoso A, Edward J D, Wamishe Y, Valent B, Wang G L, Yang Y N. The rice blast resistance gene Ptr encodes an atypical protein required for broad-spectrum disease resistance. Nat Commun, 2018, 9: 2039.

[27] 宋微. 松粳 9 号对稻瘟病抗性及抗病基因定位. 东北农业大学 硕士学位论文, 黑龙江哈尔滨, 2013.

Song W. Identification and Gene Mapping of Resistance to Magnaporthe Grisea in Songjing No. 9. MS Thesis of Northeast Agricultural University, Harbin, Heilongjiang, China, 2013 (in Chinese with English abstract).

[28] Xiao W M, Yang Q Y, Sun D Y, Wang H, Guo T, Liu Y Z, Zhu X $\mathrm{Y}$, Chen $\mathrm{Z}$ Q. Identification of three major $R$ genes responsible for broad-spectrum blast resistance in an indica rice accession. Mol Breed, 2015, 35: 49.

[29] Causse M A, Fulton T M, Cho Y G, Ahn S N, Chunwongse J, Wu K S, Xiao J H, Yu Z H, Ronald P C, Harrington S E, Second G,
McCouch S R, Tanksley S D. Saturated molecular map of the rice genome based on an interspecific backcross population. Genetics, 1994, 138: 1251-1274.

[30] Lee S, Wamishe Y, Jia Y, Liu G, Jia M H. Identification of two major resistance genes against race IE-1k of Magnaporthe oryzae the indica rice cultivar Zhe 733. Mol Breed, 2009, 24: 127-134.

[31] Sallaud C, Lorieux M, Roumen E, Tharreau D, Berruyer R, Svestasrani P, Garsmeur O, Ghesquiere A, Notteghem J L. Identification of five new blast resistance genes in the highly blast-resistant rice variety IR64 using a QTL mapping strategy. Theor Appl Genet, 2003, 106: 794-803.

[32] Zheng K L, Zhuang J Y, Lu J, Qian H R, Lin H X. Identification of DNA markers tightly linked to blast resistance genes in rice. In: Khush G S, Hettel G, Rola T, eds. Rice Genetics III (in Part 2), IRRI, Manila, Philippines, 2008. pp 565-569.

[33] Naqvi N I, Chattoo B B. Molecular genetic analysis and sequence characterized amplified region-assisted selection of blast resistance in rice. Rice Genet, 1996, 3: 570-576.

[34] Koide Y, Telebanco-Yanoria M J, Pena F D, Fukuta Y, Kobayashi N. Characterization of rice blast isolates by the differential system and their application for mapping a resistance gene, $P i 19(t)$. J Phytopathol, 2011, 159: 85-93.

[35] Li W, Lei C L, Cheng Z J, Jia Y L, Huang D L, Wang J L, Wang J K, Zhang X, Su N, Guo X P, Zhai H Q, Wan J M. Identification of SSR markers for a broad-spectrum blast resistance gene Pi20(t) for marker-assisted breeding. Mol Breed, 2008, 22: 141-149.

[36] Kumar P, Pathania S, Katoch P, Sharma T R, Plaha P, Rathour R. Genetic and physical mapping of blast resistance gene $P i-42(t)$ on the short arm of rice chromosome 12. Mol Breed, 2010, 25: 217-228.

[37] Yu Z H, Mackill D J, Bonman J M, McCouch S R, Guiderdoni E, Notteghem J L, Tanksley S D. Molecular mapping of genes for resistance to rice blast (Pyricularia grisea Sacc.). Theor Appl Genet, 1996, 93: 859-863.

[38] Hayashi K, Yoshida H, Ashikawa I. Development of PCR-based allele-specific and InDel marker sets for nine rice blast resistance genes. Theor Appl Genet, 2006, 113: 251-260.

[39] Joshi S, Dhatwalia S, Kaachra A, Sharma K D, Rathour R. Genetic and physical mapping of a new rice blast resistance specificity Pi-67 from a broad spectrum resistant genotype Tetep. Euphytica, 2019, 215: 9.

[40] Liu X Q, Yang Q Z, Lin F, Hua L X, Wang C T, Wang L, Pan Q H. Identification and fine mapping of $P i 39(t)$, a major gene conferring the broad-spectrum resistance to Magnaporthe oryzae. Mol Genet Genom, 2007, 278: 403-410.

[41] Koide Y, Telebanco-Yanoria M J, Fukuta Y, Kobayashi N. Detection of novel blast resistance genes, $P i 58(t)$ and Pi59(t), in a Myanmar rice landrace based on a standard differential system. Mol Breed, 2013, 32: 241-252.

[42] Dong L Y, Liu S F, Xu P, Deng W, Li X D, Tharreau D, Li J, Zhou J W, Wang Q, Tao D Y, Yang Q Z. Fine mapping of Pi57(t) conferring broad spectrum resistance against Magnaporthe oryzae in introgressionline IL-E1454 derived from Oryza longistaminata. PLoS One, 2017, 12: $\mathrm{e} 0186201$.

[43] Liang Z J, Wang L, Pan Q H. A new recessive gene conferring 
resistance against rice blast. Rice, 2016, 9: 47.

[44] Devi S J S R, Singh K, Umakanth B, Vishalakshi B, Rao K V S, Suneel B, Sharma S K, Kadambari G K M, Prasad M S, Senguttvel P, Syamaladevi D P, Madhav M S. Identification and characterization of a large effect QTL from Oryza glumaepatula revealed $P i 68(t)$ as putative candidate gene for rice blast resistance. Rice, 2020, 13: 17.

[45] Naqvi N I, Bonman J M, Mackill D J, Nelson R J, Chattoo B B. Identifcation of RAPD markers linked to a major blast resistance gene in rice. Mol Breed, 1995, 1: 341-348.

[46] Ahn S N, Kim Y K, Hong H C, Han S S, Choi H C, McCouch S $\mathrm{R}$, Moon H P. Mapping of genes conferring resistance to Korean isolates of rice blast fungus using DNA markers. Korean J Breed. 1997, 29: 416-423.

[47] 鲁清. 水稻种质资源重要农艺性状的全基因组关联分析. 中 国农业科学院博士学位论文, 北京, 2016.

Lu Q. Genome-wide Association Studies of Important Agronomic Traits in Rice Germplasm. PhD Dissertation of Chinese Academy of Agricultural Sciences, Beijing, China, 2016 (in Chinese with English abstract).

[48] Bagnaresi P, Biselli C, Orrù L, Urso S, Crispino L, Abbruscato P, Piffanelli P, Lupotto E, Cattivelli L, Vale G. Comparative transcriptome profiling of the early response to Magnaporthe oryzae in durable resistant vs susceptible rice (Oryza sativa L.) genotypes. PLoS One, 2012, 7: e51609.

[49] Meng Q F, Gupta R, Kwon S J, Wang Y M. Agrawal G K, Rakwal R, Park S R, Kim S T. Transcriptomic analysis of Oryza sativa leaves reveals key changes in response to Magnaporthe oryzae MSP1. Plant Pathol J, 2018, 34: 257.

[50] Li W T, Zhu Z W, Chern M, Yin J J, Yang C, Ran L, Cheng M P, He M, Wang K, Wang J, Zhou X G, Zhu X B, Chen Z X, Wang J C, Zhao W, Ma B T, Qin P, Chen W L, Wang Y P, Liu J L, Wang W M, Wu X J, Li P, Wang J R, Zhu L H, Li S G, Chen X W. A natural allele of a transcription factor in rice confers broad-spectrum blast resistance. Cell, 2017, 170: $114-126$. 